\title{
EDITORIAL
}

\section{Giving microbial diversity a home}

\author{
Efforts to house environmental microbial culture collections, resources and data are lagging \\ behind the speed of discovery in the scientific community. Emerson and Wilson explain the \\ need to increase the investment in biological resource centres to meet this growing challenge.
}

The field of environmental microbiology is a robust area encompassing research on the genetics, physiology, biochemistry, ecology and applications of microorganisms, including viruses, bacteria, archaea and micro-algae. Major advances in our understanding of environmental microbiology occur with regularity. For example, we now know that $50 \%$ of the world's oxygen supply comes from photosynthesis that is carried out by microscopic marine algae and that anaerobic oxidation of methane and ammonia by microorganisms is of global importance. In addition, we now appreciate that the high concentrations of viruses in sea water have fundamental roles in the dynamics of microbial ecosystems.

The discovery of the evolutionary and metabolic diversity of microorganisms underpins much of this research but leaves open the question of what is being done to preserve this diversity and make it accessible to the scientific community. At present, culture collections tend to be organism-based (rather than ecosystembased) and represent only a fraction of the true environmental diversity of microorganisms. To facilitate the expansion and improve the accessibility of such collections, biological resource centres (BRCs) must enable the preservation of microorganisms as well as the associated research materials and data. In the field of environmental microbiology, be it terrestrial or marine, there is a growing gap between the efforts of the research community and our capacity to maintain these resources in BRCs.

The primary mission of a $\mathrm{BRC}$ is to enable sharing of biological resources and information that promotes scientific discovery. Microbial culture collections have traditionally acted as repositories for well-characterized microorganisms that either represent new species or have unique properties that are of practical value. Most modern culture collections have expanded to become more wide-ranging BRCs that provide additional products and services such as commonly used media, bulk cultures, cell derivatives and, in some cases, identification services. The Provasoli-Guillard National Center for Culture of Marine Phytoplankton (CCMP) is a good example. It serves as a central repository to receive, maintain and distribute living cultures of marine phytoplankton, and in recent years it has extended its services to include the sale of growth media kits. The collection will expand over the next year to include marine bacteria and viruses.

The horizon holds new opportunities for BRCs. As researchers expand the number of model organisms and develop molecular tools for their analysis, BRCs can become reagent repositories. Powerful new singlecell technologies can generate enormous amounts of material (for example, from single-cell sorts) as well as downstream data, including both single-gene and whole-genome information. It will be a challenge to develop new storage methods to capture this physical output, make it available to the scientific community and couple it to the different 'omics'-type data that derive from it. The coming revolution in synthetic biology will offer a new set of challenges in both documentation and security for BRCs. Underlying all of this research in the environmental field is the need for the development of better standards, be they new strains or species, or new phylogenetic or functional panels of organisms or genes, to help validate the monitoring and testing procedures.

As the research endeavours that would benefit from the services of BRCs expand at ever increasing rates, their continued support, as well as opportunities for developing new BRCs, is a real challenge. Some countries, notably Germany and Japan, have appreciable governmental support for microbial BRCs such as the DSMZ (known in English as the German Collection of Microorganisms and Cell Cultures) and the Japan Collection of Microorganisms (JCM); however, these countries are the exceptions. In the United States, government expenditure for the support of environmental microbial BRCs is probably at an alltime low. By comparison, in areas of biomedical microbiology there are large programmes aimed at specialty collections of the microorganisms that are of concern to human health, funded primarily through the US National Institutes of Health.

As environmental microbiology enters the era of petabyte science it will be crucial that the raw materials that drive this research have provenance, documentation and a home. The continued support and development of BRCs to carry out these tasks is essential, just as it is important that BRCs themselves respond to the challenges of these new frontiers to serve the scientific community. 